\title{
Modelamiento molecular de la interacción de ibuprofeno con las enzimas Ciclooxigenasa 1, 2 y el Citocromo P450 2 C9
}

\author{
Lorena Meneses $\mathrm{O}^{1}$, María Fernanda Pilaquinga $\mathrm{F}^{1}$ y Sebastián Cuesta $\mathrm{H}^{1}$ \\ ${ }^{1}$ Laboratotio de Química Computacional, Facultad de Ciencias Exactas y Naturales, Pontificia Universidad Católica del \\ Ecuador, Quito, Ecuador
}

1mmeneses@puce.edu.ec

Recibido: 2014-06-20; aceptado: 2014-10-10

\section{RESUMEN.-}

En esta investigación, se presenta el modelamiento computacional de la interacción de ibuprofeno con las enzimas Ciclooxigenasa 1, Ciclooxigenasa 2 y Citocromo P450 2C9. El objetivo fue comprobar la aplicabilidad de métodos de acoplamiento molecular en la determinación de nuevos ligandos y la localización de sitios activos en las enzimas, así como también tener un mejor entendimiento del mecanismo de acción farmacológica de ibuprofeno. Para el estudio se aplicaron métodos de dinámica molecular, para modelar la interacción de la molécula de ibuprofeno con las enzimas, determinando el sitio activo de éstas. Se utilizaron los programas Autodock 4 y Autodock VINA. En el modelamiento molecular, los mejores resultados se lograron con el programa Autodock VINA, por lo que éstos fueron comparados con resultados experimentales obtenidos mediante cristalografía de rayos $\mathrm{X}$. Los métodos computacionales de acoplamiento molecular son totalmente comparables con resultados obtenidos experimentalmente, demostrando ser bastante exactos. Esto comprueba la aplicabilidad de estos métodos en el proceso de síntesis y diseño de nuevos fármacos.

PALABRAS CLAVES: acoplamiento, autodock, ciclooxigenasa, citocromo P450, ibuprofeno

\section{ABSTRACT.-}

In this research, a computational modeling of the interaction of ibuprofen with the enzymes Cyclooxygenase 1, Cyclooxygenase 2 and Cytochrome P450 2C9 is presented. The aim of this study was to test the applicability of molecular docking methods in the identification of new ligands and the location of active sites in enzymes; also to have a better understanding of the action mechanism of ibuprofen. The research encompasses modeling the interaction of the ibuprofen molecule with the enzymes through molecular dynamics methods. AutoDock 4 and AutoDock VINA software were used. In the molecular modeling, the best results were achieved with AutoDock VINA, so those results were compared with experimental data obtained by X-ray crystallography. Computational docking methods are fully comparable with experimental results proving to be quite accurate. This demonstrates the applicability of these methods in the synthesis and design of new drugs.

KEYWORDS: autodock, cyclooxygenase, cytochrome P450, docking, ibuprofen 


\section{INTRODUCCIÓN}

El proceso inflamatorio es la respuesta a un estímulo nocivo causado por infecciones, anticuerpos o lesiones físicas. Está constituido por una serie de fenómenos moleculares, celulares y vasculares (Brunton y Parker, 2008). La habilidad de tener una respuesta inflamatoria es esencial para sobrevivir al ataque de agentes dañinos. Como resultado del proceso inflamatorio, el umbral del dolor se reduce y el área afectada se hace más sensible. Este proceso hace que exista menor contacto y, por ende, menor movimiento del área afectada, promoviendo la sanación (DiPiro y Talbert, 2008).

El ser humano siempre ha buscado alivio para el dolor. El uso de sustancias medicinales para tratar el dolor y la fiebre data de las antiguas civilizaciones griegas y egipcias, donde las hojas de mirto seco, o extractos amargos de la corteza de los árboles de álamo, se utilizaban para tratar dolores abdominales y de espalda (Hall et.al, 2001). Hoy en día, el impacto del dolor en la sociedad es todavía grande, siendo la principal razón para buscar ayuda médica.

El ibuprofeno es uno de los antiinflamatorios no esteroideos (AINE's) más usados en la actualidad. Fue creado por el grupo de investigadores de la compañía Boots en 1960 (Lednicer, 2008), demostrando ser más efectivo que sus predecesores y, que a su vez, causa menos efectos secundarios. El ibuprofeno pertenece a la familia de los derivados del ácido propiónico. Posee acción analgésica, antipirética y antiinflamatoria. El ibuprofeno es uno de los medicamentos antiinflamatorios catalogados por la Organización Mundial de la Salud (OMS) como esenciales.

El ibuprofeno es un inhibidor no selectivo de la Ciclooxigenasa (COX-1 y COX-2). La COX-1 participa en la producción de prostaglandinas que intervienen en procesos fisiológicos tales como protección del epitelio gástrico, mantenimiento del flujo renal, agregación plaquetaria, migración de neutrófilos, entre otros. La COX-2 produce prostaglandinas adicionales que intervienen y causan los efectos en un proceso infla- matorio. Su función es mediar en los procesos de inflamación. Es parte fundamental en el sistema nervioso central y el riñón. No es constitutiva en todos los tejidos, pero puede ser inducida en el sitio de inflamación, manteniendo los mecanismos inflamatorios y amplificando las señales dolorosas (Hall et.al., 2001).

Gracias a métodos instrumentales como la cristalografía de rayos $\mathrm{X}$, hoy se puede conocer la estructura tridimensional de biomoléculas, y la forma en que ciertos medicamentos se acoplan a ellas. El conocimiento de propiedades físicas, químicas y farmacológicas de principios activos es de mucha utilidad industrial, ya que permiten entender el comportamiento químico en el cuerpo y el mecanismo de acción. Con un estudio computacional completo de estas sustancias, se pueden crear procesos de síntesis más eficientes, menos costosos o más sencillos; y además se pueden sintetizar nuevos fármacos de acción rápida y que produzcan menos efectos secundarios. Esto repercute en el costo de los medicamentos, que puede disminuir notablemente y llegar a personas de escasos recursos (WHO, 2010).

El propósito de la química computacional es la determinación de propiedades químicas de interés, así como el desarrollo de compuestos novedosos y el mejoramiento de procesos de síntesis. La química computacional elimina en gran medida costos de experimentación y tiempo. Con cálculos computacionales, se puede modelar un sistema molecular antes de sintetizar una molécula en el laboratorio; $y$, aunque no son perfectos, son bastante buenos para descartar el $90 \%$ de posibles compuestos que son inadecuados para su uso. Esto es bastante útil, ya que la síntesis de un solo compuesto puede tomar meses, mucha materia prima y generar desechos tóxicos (Ramachandran, 2008; Young, 2001). Las técnicas de modelamiento molecular son ampliamente usadas en industrias químicas, farmacéuticas y agroquímicas. En ningún caso, la aplicación de la química computacional sustituye la experimentación en el laboratorio, sino que sirven como una herramienta versátil para estudiar el funcionamiento de la materia. 
El diseño racional de medicamentos, es el proceso de encontrar nuevos compuestos, basados en el conocimiento del objetivo biológico. Un fármaco es comúnmente una molécula orgánica pequeña que activa o inhibe el funcionamiento de una biomolécula, resultando en un beneficio terapéutico para el paciente. Utilizando una amplia gama de software y estructuras computarizadas de referencia, se puede determinar las moléculas que podrían bloquear cierto tipo de enzima. Estas moléculas pueden ser obtenidas de un banco de moléculas, o modeladas en la computadora y luego sintetizadas en laboratorio. Durante el proceso de desarrollo normal, aproximadamente el $40-60 \%$ de los compuestos son rechazados debido a sus propiedades ADMET (absorción correlativa, distribución, metabolismo, excreción y toxicidad) no favorables (Rahman et.al., 2009). La mayor parte de los esfuerzos en modelamiento molecular se los realiza para predecir las propiedades ADMET, ya que es una manera económica y de alto rendimiento de evaluar estas propiedades, antes de la síntesis y las pruebas biológicas. Las predicciones ADMET pueden lograr el rechazo de un prototipo por su baja biodisponibilidad oral, recién iniciado el proceso. Este método de diseño de fármacos acorta el tiempo de invención de un medicamento y ahorra millones de dólares a las compañías, en comparación al desarrollo tradicional de fármacos. Hoy en día, toma menos de 8 años poner 3 medicamentos contra el VIH en el mercado, cuando normalmente tomaba el doble, y a costos excesivos (National Institute of General Medical Sciences, 2011; Mandal y Moudgil, 2009).

El programa computacional Autodock, está diseñado como una herramienta de acoplamiento molecular (The Scripps Research Institute, 2013). Autodock trata de predecir la estructura o estructuras de los complejos intermoleculares formados entre dos o más moléculas. Docking, como también se lo llama, es ampliamente usado para sugerir enlaces e inhibir proteínas. Con este programa, se pueden predecir cómo moléculas pequeñas, sustratos y candidatos de fármacos, se unen a estructuras tridimensionales conocidas (The Scripps Research Institute, 2013). Así mismo, AutoDock puede ser aplicado en diseños de fármacos, optimización de compuestos líder, estudios en mecanismos químicos, cristalografía de rayos $X$, entre otros. El programa AutoDock consiste en dos generaciones de programas para computadora: AutoDock 4 y AutoDock Vina (Atkinson y Abernethy, et al., 2007).

La aplicación de programas computacionales en el estudio del acoplamiento de ibuprofeno con las Ciclooxigenasas 1 y 2 y con el Citocromo P450, ha permitido comprobar su utilidad en la determinación de nuevos ligandos y en la localización de sitios activos en las enzimas, así como también tener un mejor entendimiento del mecanismo de acción farmacológica del ibuprofeno.

\section{MATERIALES Y MÉTODOS}

Para el modelamiento computacional de la interacción de ibuprofeno con las enzimas COX-1, COX-2 y Citocromo P450 2C9 (CYP2C9), se utilizaron los programas Autodock $4 \mathrm{y}$ Autodock VINA.

El ibuprofeno se une a las enzimas COX-1 y COX-2 para ejercer su acción, y a el CYP2C9 para su metabolización. En la Figura 1 se encuentran las estructuras tridimensionales de la COX-1, COX-2 y CYP2C9. Las enzimas fueron obtenidas experimentalmente mediante la técnica de rayos $\mathrm{X}$ por otros autores, $\mathrm{y}$ colocadas en un banco de proteínas (Protein Data Banks), de donde se las descargó. 


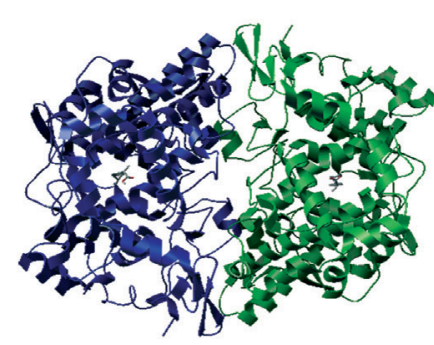

Estructura COX1

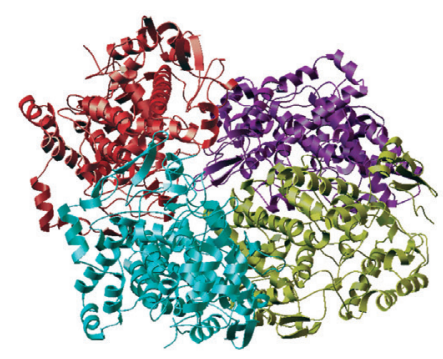

Estructura COX2

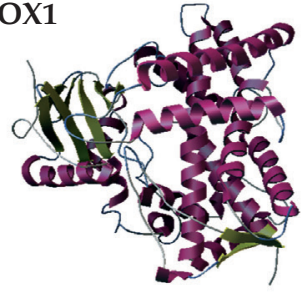

Estructura CYP2C9

Figura 1. Estructuras de las enzimas Ciclooxigensa 1 y 2 (COX-1 y COX-2) y citocromo P450 2C9 (CYP2C9) obtenidas de las base de datos Protein Data Banks

Para el modelamiento, se hizo interactuar al ligando (el ibuprofeno) con la macromolécula (las enzimas). Se determinaron los posibles sitios activos de la enzima que son los que tienen una menor energía (mayor afinidad). El programa Autodock permite seleccionar, para el cálculo de acoplamiento molecular, cualquier zona de la enzima. Esto sirve cuando se tiene una idea de en qué zona de la enzima se encuentra el sitio activo. En este estudio, se seleccionó toda la enzima.

El procedimiento que se siguió para las tres enzimas fue el siguiente: Se obtuvieron las enzimas del banco de datos de proteínas en formato PDB.

Utilizando el programa Chimera 1.7 (Pettersen et.al., 2004), se determinaron todos los residuos que no pertenecían a la proteína.

En Autodock, se abrió Autodock Tools, se escogió la enzima como macromolécula y se la limpió con la opción Hydrogen Polar only. Posteriormente, se escogió al ibuprofeno como ligando y se estableció el área de acoplamiento, como se muestra en la Tabla 1, donde se indican las condiciones del cálculo.

Tabla 1. Condiciones y áreas de análisis para el acoplamiento

\begin{tabular}{cccc}
\hline Parámetro & COX-1 & COX-2 & CYP2C9 \\
\hline Centro X & 47.112 & 53.975 & 8.093 \\
Centro Y & 28.000 & -16.705 & 32.809 \\
Centro Z & 194.758 & -12.800 & 0.802 \\
Tamaño X & 48 & 60 & 20 \\
Tamaño Y & 20 & 20 & 20 \\
Tamaño Z & 20 & 20 & 20 \\
\hline
\end{tabular}


Utilizando Autodock 4, se realizó el cálculo de acoplamiento molecular entre la macromolécula y el ibuprofeno. Finalmente, con los datos obtenidos en Autodock Tools, se creó un archivo

\section{RESULTADOS}

Al realizar el cálculo de modelamiento molecular del acoplamiento de ibuprofeno con la COX-1, el programa Autodock 4 muestra diez resultados diferentes. Cada resultado representa al ligando en una posición espacial distinta. En cada posi- de entrada para Autodock VINA y se realizó el cálculo con este programa. Los resultados se visualizaron utilizando el programa PyMol.

ción se obtiene la afinidad entre la macromolécula y el ligando. En la Tabla 2 se presentan las diez conformaciones obtenidas del acoplamiento del ibuprofeno con la COX-1.

Tabla 2. Conformaciones obtenidas del cálculo de acoplamiento de Ibuprofeno con COX-1, COX2 y CYP2C9, con los valores de afinidad en $\mathrm{kcal} / \mathrm{mol}$, utilizando los programas Autodock 4 y Autodock VINA

\begin{tabular}{ccccc} 
Conformación & \multicolumn{4}{c}{ Afinidad (kcal/mol) } \\
\cline { 2 - 5 } & COX-1 Autodock 4 & $\begin{array}{c}\text { COX-1 Autodock } \\
\text { VINA }\end{array}$ & $\begin{array}{c}\text { COX-2 Autodock } \\
\text { VINA }\end{array}$ & $\begin{array}{c}\text { CYP2C9 Autodock } \\
\text { VINA }\end{array}$ \\
\hline 1 & -3.97 & -7.6 & -7.4 & -7.6 \\
2 & -3.68 & -7.6 & -7.4 & -6.8 \\
3 & -3.76 & -7.4 & -7.4 & -6.8 \\
4 & -4.78 & -7.3 & -7.3 & -6.8 \\
5 & -3.39 & -7.3 & -6.9 & -6.7 \\
6 & -5.09 & -7.2 & -6.9 & -6.1 \\
7 & -4.62 & -7.1 & -6.9 & -6.0 \\
8 & -3.86 & -6.7 & -6.9 & -5.8 \\
9 & -4.51 & & -6.8 & -5.8 \\
10 & -3.87 & & &
\end{tabular}

Las conformaciones 4 y la 6 son las que tienen mayor afinidad por lo que son las conformaciones más probables de la interacción. En la Figura 2A, se muestra la posición en el espacio de estas dos conformaciones dentro de la enzima. En la parte izquierda se presenta la posición espacial del ibuprofeno dentro de la COX-1 para la conformación 4, mientras que en la parte derecha se presenta la posición para la conformación 6 . En la Figura 2B, se presenta la interacción entre ibuprofeno y COX-1 obtenida experimentalmente por cristalografía de rayos X. La enzima muestra los dos sitios activos donde se ubica el ibuprofeno, pero también muestra otras estructuras como glucósidos, agua y el grupo hemo (Protoporfirina IX) que funciona como cofactor de la enzima. Se comparó la estructura obtenida del modelamiento computacional, con la estructura experimental. En la Figura 2C, la molécula experimental se muestra en color gris, mientras que la molécula en celeste representa la calculada computacionalmente. 
A

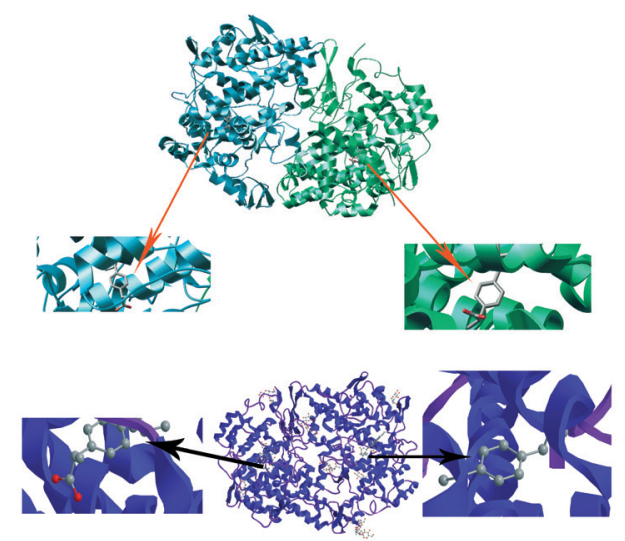

C

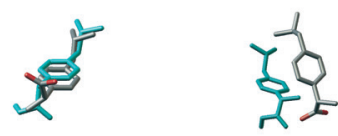

Figura 2. A) Disposición espacial del ligando en la conformación 4 (izq.) y 6 (der.); B) Estructura experimental de acoplamiento Ibuprofeno COX-1; C) Comparación de las conformaciones de Ibuprofeno obtenidos experimentalmente (gris) y computacionalmente (celeste) para las conformaciones 4 (zq.) y 6 (der.) con el programa Autodock 4

Se realizó el mismo cálculo utilizandoe el programa Autodock VINA, con el que se obtuvieron mejores resultados, ya que se encontraron mayores afinidades de acoplamiento, lo que implica menores energías de enlace, como se observa en la Tabla 2. El programa Autodock VINA muestra las conformaciones ordenadas energéticamente de menor a mayor, por lo que las conformaciones 1 y 2 son las de menor energía, debido a su alta afinidad.
En la Figura 3A, la conformación 1 se presenta en color verde mientras la conformación 2 se presenta en color rojo, la molécula experimental se muestra de color gris. El modelamiento molecular finaliza al acoplar las conformaciones obtenidas computacionalmente dentro de la enzima, como se muestra en la Figura 3B.

A
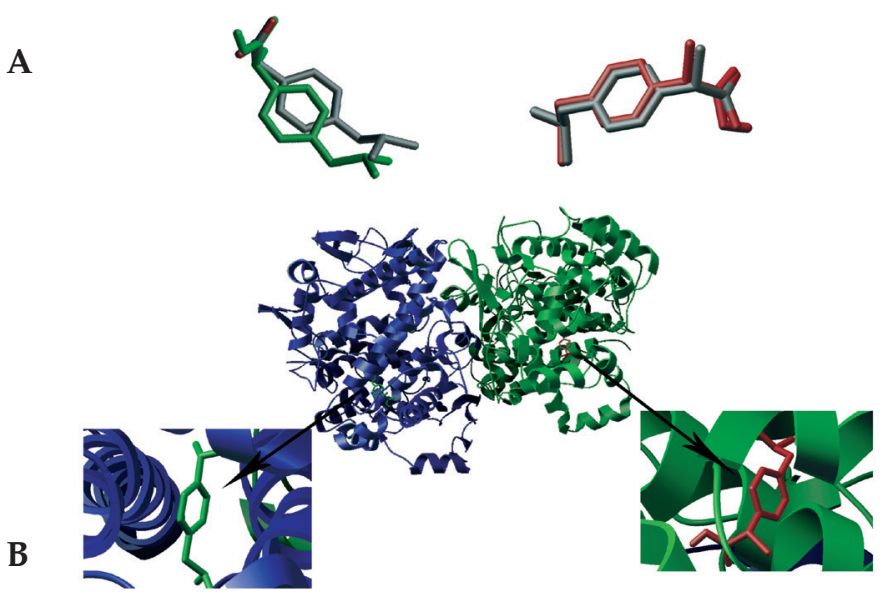

Figura 3. A) Comparación de las conformaciones de Ibuprofeno obtenidas experimentalmente (gris) y computacionalmente para las conformaciones 1 (izq. verde) y 2 (der. rojo) con el programa Autodock VINA; B) Estructura computacional de acoplamiento Ibuprofeno COX-1 
Debido a que se obtuvieron mejores resultados con Autodock VINA, sólo se mostrarán los acoplamientos calculados con este programa. En la Tabla 2 se muestran las afinidades de las conformaciones obtenidas de la interacción del ibuprofeno con la COX-2.

Se tomaron las tres primeras conformaciones ya que son las de mayor afinidad. Las dos primeras se encuentran en el mismo sitio activo de la enzima mientras la conformación
3 se encuentra en el otro sitio activo. A continuación se compararon las conformaciones obtenidas computacionalmente, con un modelo experimental de la interacción entre la COX-2 del ratón común con el medicamento celecoxib. El resultado la comparación se presenta en la Figura 4A. Para presentar el resultado final del acoplamiento, se tomaron las conformaciones 1 y 3, que son las más probables para cada sitio activo. El acoplamiento se puede observar en la Figura 4B.

A
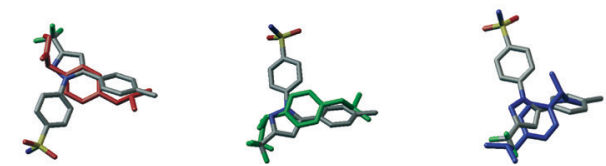

B

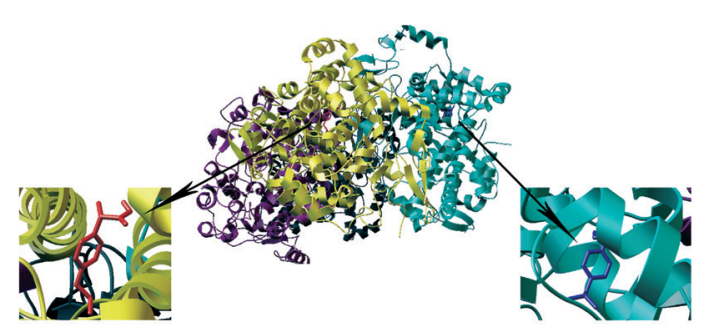

Figura 4. A) Comparación de las conformaciones de celecoxib obtenidas experimentalmente (gris) con el ibuprofeno obtenido computacionalmente para las conformaciones 1 (izq. rojo), 2 (medio, verde) y 3 (der. azul); B) Estructura computacional de acoplamiento Ibuprofeno COX-2

El último acoplamiento se realizó entre el CYP2C9 y el ibuprofeno. En la Tabla 2 se encuentran las conformaciones obtenidas y los valores de afinidad.

Se tomó la conformación con la mayor afinidad y se la comparó con un modelo experimental de la interacción entre la CYP2C9 de un humano con un AINE derivado del ácido propiónico, el flurbiprofeno. En la Figura 5A se muestra el resultado de la comparación. En la Figura 5B se muestra el resultado final del acoplamiento molecular del ibuprofeno con el CYP2C9.

A
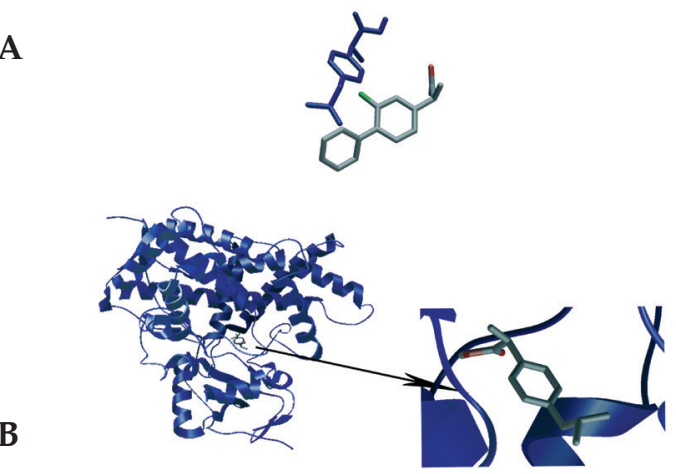

Figura 5. A) Comparación de la conformación de flurbiprofeno obtenida experimentalmente (gris) con el ibuprofeno obtenido computacionalmente para la conformación 1 (azul); B) Estructura computacional de acoplamiento Ibuprofeno CYP2C9 


\section{DISCUSIÓN}

Estudios previos demuestran que las enzimas Ciclooxigenasa poseen dos sitios activos en su estructura (DeWitt, 1999). En el modelamiento computacional realizado en este trabajo, se ha observado que las conformaciones de mayor afinidad del ibuprofeno, se ubican en la parte inferior de las enzimas COX-1 y COX-2, en dos lugares diferentes, que corresponden a los dos sitios activos ciclooxigenasa que poseen estas enzimas. Este resultado demuestra que el acoplamiento molecular realizado con el modelamiento computacional es acertado, pues reproduce adecuadamente los sitios activos de las enzimas ciclooxigenasa, que interactúan con el ibuprofeno.

La conformaciones de mayor afinidad obtenidas computacionalmente para el ibuprofeno, utilizando el programa Autodock 4, son la conformación 4 y 6 (Tabla 2). Estas conformaciones se compararon con las obtenidas experimentalmente para la COX-1. Se puede observar en la figura $2 \mathrm{C}$, que en la conformación 4, ambas moléculas están casi superpuestas, variando muy poco en la posición; mientras que en la conformación 6, las moléculas están ligeramente separadas. Esto nos demuestra que los resultados obtenidos con el programa Autodock 4 no son exactamente iguales a los experimentales, pero si lo suficientemente cercanos, como para guiarnos sobre cuáles serían las conformaciones más adecuadas.

Al realizar el mismo estudio pero utilizando el programa Autodock VINA, se obtienen conformaciones con afinidades más altas (Tabla 2), lo que se traduce en menor energía de enlace. Al comparar los valores de las afinidades obtenidos con los dos programas, se puede ver que al utilizar el programa Autodock VINA, las afinidades son mayores en casi $2 \mathrm{kcal} / \mathrm{mol}$, lo cual crea conformaciones mucho más exactas, al compararlas con los datos experimentales, como se puede visualizar en la Figura 3A. En este caso se observa que ambas estructuras están superpuestas casi perfectamente. La conformación 1 y la 2 poseen la misma afinidad, lo que indica que tanto el sitio activo de la derecha como el de la izquierda de la COX-1, tienen la misma probabilidad para que ingrese el ibuprofeno y ejerza su función.

$\mathrm{Al}$ introducir el ibuprofeno dentro de la enzima COX-1, se puede observar además, que la figura 3B, donde se presenta el acoplamiento molecular computacional, es muy similar a la figura 2B, donde se presenta el acoplamiento experimental obtenido por cristalografía de rayos $X$, lo que demuestra la eficiencia de los métodos computacionales, y que el modelamiento computacional permite obtener resultados comparables con los experimentales.

En la tabla 2 se presentan las nueve conformaciones obtenidas para el acoplamiento del Ibuprofeno con la COX-2 con su respectiva afinidad. En esta tabla se puede observar que las tres primeras conformaciones son las de mayor afinidad, por lo tanto son las que presentarán menores energías de enlace con la enzima. Las dos primeras conformaciones se encuentran en el mismo sitio activo de la enzima, mientras que la conformación 3 se encuentra en el otro sitio activo. En el caso de la interacción del ibuprofeno con la COX-2, la molécula de ibuprofeno fue comparada con la conformación del medicamento celecoxib obtenida experimentalmente por cristalografía de rayos X (Figura 4A). El celecoxib es un AINE selectivo de la COX-2, por lo que, aunque la forma de unirse a la enzima es diferente, el sitio activo donde se une es el mismo que el del ibuprofeno. Las tres conformaciones se encuentran superpuestas a la molécula de celecoxib, lo que indica que los dos sitios activos encontrados para la COX-2, son correctos, y concuerdan con los encontrados en trabajos previos (DeWitt, 1999).

En el caso la interacción del ibuprofeno con el citocromo P450 2C9, se puede observar en la tabla 2, que existe una sola conformación con la mayor afinidad. Esta conformación fue comparada con una molécula de fluriprofeno obtenida experimentalmente (Figura 5A). En este caso, las moléculas no se encuentran superpuestas, aunque si están muy cercanas una a otra, lo que indica que el sitio activo encontrado com- 
putacionalmente, es el mismo que el encontrado experimentalmente. Así, este sitio es donde se producirá la hidroxilación del grupo isobutilo del ibuprofeno, para proceder a la formación de otros metabolitos y su excreción renal.

Las comparaciones analizadas son muy útiles, para determinar la aplicabilidad y exactitud de los resultados obtenidos mediante programas computacionales, en la búsqueda de sitios activos y de enlaces enzima-sustrato de objetivos biológicos y enzimas de interés.

La diversidad de mecanismos de enlace de la COX-1 y COX-2 demostrados para diferentes compuestos es impresionante. La habilidad de la enzima de acomodarse estructuralmente a diferentes inhibidores es notable, ya que no existe evidencia de cambios considerables en la conformación de las proteínas, en la interacción con AINEs (Blobaum y Marnett, 2007). El ibuprofeno, como mostraron los resultados, bloquea el sitio activo ciclooxigenasa de la enzima (impidiendo la formación de la prostaglandina). El mensaje normal no es entregado, por lo que no se siente el dolor y no se activa la respuesta inflamatoria.

Desafortunadamente, al ser un medicamento no selectivo, ambas isoenzimas son bloqueadas, interrumpiendo no solo la respuesta inflamatoria, sino otras funciones protectoras, como la producción de ácido en el estómago (McMurry, 2004). Esta inhibición se da por competencia. La inhibición competitiva se produce cuando una molécula, que se asemeja al sustrato, puede ocupar el sitio catalítico debido a su similitud en estructura, siendo parcial o completamente inactivo. Al ocupar el sitio activo, esta molécula actúa como un inhibidor competitivo, previniendo que el sustrato normal sea examinado y catalizado. Operacionalmente, inhibidores competitivos se unen reversiblemente al sitio activo. La inhibición puede ser revertida al diluir el inhibidor o aumentando la cantidad de sustrato (Tinoco et al., 2009).

Luego de ejercer su función de inhibir la enzima, el ibuprofeno pasa al hígado, para ser metabolizado por la enzima citocromo P450 monooxi- genasa $2 \mathrm{C}$, en un proceso de hidroxilación en el carbono terciario del isobutilo, aumentando su solubilidad en agua para poder ser eliminado por vía renal.

\section{CONCLUSIONES}

- El estudio de modelamiento computacional del ibuprofeno nos ha indicado que es un inhibidor no selectivo de la ciclooxigenasa, al unirse a ambas isoformas de manera indistinta.

- El ibuprofeno se une sólo a los sitios activos de la ciclooxigenasa en la parte inferior de la enzima.

- Los métodos computacionales de aclopamiento molecular son totalmente comparables con los resulados obtenidos experimentalmente, demostando ser bastante precisos. Esto demuestra la aplicabilidad de estos métodos en el proceso de síntesis y diseño de nuevos fármacos.

\section{AGRADECIMIENTO}

A la DGA-PUCE, a través del proyecto "Estudio téorico y experimental en síntesis orgánica (Ibuprofeno)", con código J13075.

\section{REFERENCIAS BIBLIOGRÁFICAS}

Atkinson A y Abernethy D. 2007. Principles of Clinical Pharmacology. Segunda edición. Elsevier Inc. UK. 146, 149 pp.

Blobaum A y Marnett L. 2007. Structural and Functional Basis of Cyclooxygenase Inhibition, Journal of Medicinal Chemistry, 50(7): 1425-1441.

Brunton L y Parker K. 2008. Manual of Pharmacology and Therapeutics. McGraw-Hill. USA. 428-432 pp.

DeWitt D. 1999. Cox-2-Selective Inhibitors: The new super aspitin. Molecular Pharmacology, 55: 625-631. 
DiPiro J y Talbert R. 2008. Pharmacotherapy: A Pathophysiologic Approach. Séptima edición. McGraw-Hill. USA. 989, 990 pp.

Hall V, Murillo N, Rocha M y Rodriguez E. 2001. Antiinflamatorios No Esteroidales. Centro Nacional de Información de Medicamentos. Instituto de Investigaciones Farmacéuticas. Universidad de Costa Rica. Costa Rica. 7: 13,14 pp.

Lednicer D. 2008. The organic Chemistry of Drug Synthesis. Wiley-Interscience. New Jersey. USA. 85-86 pp.

Mandal S y Moudgil M. 2009. Rational Drug Design. European Journal of Pharmacology. 625: 90-100

McMurry J. 2004. Organic Chemistry, Thomson, USA, 305, 519, 1034, 1035 pp.

National Institute of General Medical Sciences. 2011. Structure-Based Drug Design: From the Computer to the Clinic. Página de Internet: http:/ / publications.nigms.nih. gov / structlife/ chapter4.html. Consultada 25-enero-2013.

Pettersen E, Goddard T, Huang C, Couch G, Greenblatt D, Meng E, Ferrin T. 2004. UCSF Quimera - a visualization system for exploratory research and analysis. Journal of Computational Chemistry. 25 (13): 1605-1612.
Rahman A, Caldwell G, Choudhary M y Yan Z. 2009. Frontiers in Drug Design and Discovery. Bentham Science. USA. 333, 334 pp.

Ramachandran K. 2008. Computational Chemistry and Molecular Modeling Principles and Applications. Springer-Verlag. UK. 2, 115 pp.

The Scripps Research Institute. 2013. Autodock. Página de Internet: autodock.scripps. edu/ Consultada 24-septiembre-2013.

Tinoco Jr, Sauer K y Wang J. 1978. Physical Chemistry Principles and Applications in Biological Sciences, Prentice Hall, USA, 418, 435 pp.

WHO Media centre. 2010. Medicamentos: Medicamentos esenciales. Página de Internet: http: / / www.who.int/mediacentre/ factsheets / fs325/es/index.html. Consultada 25-enero-2013.

Young D. 2001. Computational Chemistry A Practical Guide for Applying Techniques to Real-World Problems. John Wiley \& Sons Inc. USA. 1, 3, 19, 86, 113 pp. 